\title{
The Theory of Strong Coupling Superconductivity in Disordered Transition Metal Alloys
}

\author{
K. I. Wysokiński \\ Institute of Physics, M. Curie-Sktodowska University, Lublin, Poland
}

A. L. Kuzemsky

Laboratory for Theoretical Physics, Joint Institute for Nuclear Research, Dubna, USRR

(Received January 3, 1983)

The equations of strong coupling superconductivity in disordered transition metal alloys have been derived by means of "irreducible" Green's functions and on the basis of the alloy version of the Barisić-Labbé-Friedel model for electron-ion interaction. The configurational averaging has been performed by means of the coherent potential approximation. Making some approximations, we have obtained the formulas for the transition temperature $T_{c}$ and the electron-phonon coupling constant $\lambda$. These depend on the alloy component and total densities of states, the phonon Green's function, and the parameters of the model.

\section{INTRODUCTION}

The modern microscopic theory of superconductivity was given a rigorous mathematical formulation in the classic work of Bogolubov and co-workers ${ }^{1-3}$ and others. ${ }^{4-8}$ It was shown that the equations of superconductivity can be derived from the fundamental electron-ion and electronelectron interactions. The set of equations obtained is known as the Eliashberg equations. They enable us to investigate the electronic and lattice properties of a metal in both the normal and superconducting states. Moreover, the Eliashberg equations are appropriate to the description of strong coupling superconductors, in contrast to the so-called Gorkov equations, which are valid in the weak coupling regime and describe the electron subsystem in the superconducting state only.

Extensions of the theory to disordered superconductors have been given for the "dirty" and dilute ${ }^{10}$ alloy limits. Interest in theoretical and experimental study of disordered superconductors has increased, ${ }^{11}$ and 
much effort has been devoted to transition metal compounds ${ }^{12}$ and substitutionally disordered alloys. ${ }^{13}$

A number of papers ${ }^{14-21}$ have described concentrated superconducting alloys, using the Gorkov weak coupling approach and the coherent potential approximation (CPA) to treat disorder. They use the following model Hamiltonian with Cooper pair sources $\Delta_{i}$ :

$$
H=\sum_{i \sigma} \epsilon_{i \sigma} n_{i \sigma}+\sum_{i j \sigma} t_{i j} a_{i \sigma}^{+} a_{j \sigma}-\sum_{i}\left(\Delta_{i} a_{i \uparrow}^{+} a_{i \downarrow}^{+}+\Delta_{i}^{*} a_{i \downarrow} a_{i \uparrow}\right)
$$

These papers discuss the influence of the disorder on the electron subsystem. The phonon-mediated parameters of the effective electron-electron interaction in alloys entering the definition of $\Delta_{i}$ in (1) have been recently derived $^{22}$ on the basis of the random contact model. On the other hand, Grünewald and Scharnberg ${ }^{23}$ studied the effect of force constant disorder on the electron-phonon spectral function $\alpha^{2}(z) F(z)$, while Appel ${ }^{24}$ investigated the influence of atomic ordering in alloys on their $T_{c}$ by means of the integral equation for the vertex part.

Eliashberg-type theories have also been proposed for superconducting alloys. ${ }^{25,26}$ Kerker and Bennemann ${ }^{25}$ used a Fröhlich-type Hamiltonian for the electron-phonon interaction and neglected the effect of disorder on the phonon Green's function (GF). Lustfeld ${ }^{26}$ obtained the expression for $T_{c}$ on the basis of a phenomenological ansatz for the averaged anomalous self-energy [cf. Eq. (16) for $\hat{\sigma}_{\text {eff }}$ in Ref. 26].

The purpose of the present paper is to develop a microscopic selfconsistent theory of strong coupling superconductivity in disordered transition metal alloys. The alloy version ${ }^{27}$ of the Barišić-Labbé-Friedel (BLF) tight-binding model ${ }^{28,29}$ is used for the electron-ion interaction. As was shown in Refs. 30-32, the BLF phonon-induced $d-d$ coupling is the dominant mechanism for superconductivity in such systems. We derive the equations for superconductivity in the site representation by means of the irreducible Green's function method ${ }^{33-35}$ in Section 2, using ideas developed previously in connection with the derivation of Eliashberg-type equations for pure transition metals in the Wannier representation. ${ }^{35}$ Various attempts at configurational averaging are discussed in Section 3, where the formula for $T_{\mathrm{c}}$ is also obtained. Section 4 contains a discussion and conclusions.

\section{GENERAL THEORY}

When studying superconductivity in transition metal alloys one must take into account at least three facts of major importance: (i) The $d$ electrons responsible for superconductivity in these systems have atomic character 
(Section 2.1); (ii) these materials usually belong to the class of strong coupling superconductors (Sections 2.2 and 2.3); (iii) they are very often disordered, so to obtain meaningful results requires the proper averaging (Section 3).

\subsection{The Hamiltonian}

In the so-called modified tight-binding method we write the Hamiltonian for a given configuration of atoms in an alloy $\mathrm{as}^{27}$

$$
H=\sum_{i \sigma} \epsilon_{i} n_{i \sigma}+\frac{1}{2} \sum_{i \sigma} U_{i} n_{i \sigma} n_{i-\sigma}+\sum_{i j \sigma}^{\prime} t_{i j} a_{i \sigma}^{+} a_{j \sigma}+H_{\mathrm{e}-\mathrm{i}}+\mathrm{H}_{\mathrm{i}}
$$

Here $n_{i \sigma}=a_{i \sigma}^{+} a_{i \sigma}$, and $a_{i \sigma}^{+}\left(a_{i \sigma}\right)$ creates (annihilates) the $d$ electron in the "Wannier" state $\psi_{i \sigma}$ with spin $\sigma$. The $t_{i j}$ are the hopping matrix elements, and the prime indicates that the sum over $j$ is limited to nearest neighbors of $i$. Here $\epsilon_{i}$ and $U_{i}$ are random "energy levels" and intrasite Coulomb matrix elements, respectively. $H_{\mathrm{e}-\mathrm{i}}$ stands for the electron-ion interaction Hamiltonian. This part of $H$ was derived previously ${ }^{27}$ and is a direct generalization of the $\mathrm{BLF}^{28}$ model,

$$
H_{\mathrm{e}-\mathrm{i}}=\sum_{i j \sigma} \sum_{\alpha} T_{i j}^{\alpha}\left(u_{i}^{\alpha}-u_{j}^{\alpha}\right) a_{i \sigma}^{+} a_{j \sigma}
$$

with

$$
T_{i j}^{\alpha}=\frac{q_{0}^{i}+q_{0}^{j}}{2} t_{i j} \frac{R_{j}^{\alpha}-R_{i}^{\alpha}}{\left|\mathbf{R}_{j}-\mathbf{R}_{i}\right|}
$$

Here $u_{i}^{\alpha}$ is the $\alpha$ th component of the displacement of an ion at the $i$ th site, and $q_{0}^{i}$ is the Slater coefficient describing an exponential [exp $\left(-q_{0}^{i} r\right)$ ] decrease of the $d$-electron wave function. ${ }^{28}$ It takes on the value $q^{\mathrm{A}}\left(q^{\mathrm{B}}\right)$ when the atom at site $i$ is of $\mathrm{A}$ (B) type. $\mathbf{R}_{j}-\mathbf{R}_{i}=\mathbf{R}_{j i}$ is the relative position vector of two ions at $i$ and $j$. The last part of the Hamiltonian represents the ion subsystem and in the harmonic approximation used here it is given by

$$
H_{\mathrm{i}}=\sum_{i} \frac{p_{i}^{2}}{2 M_{i}}+\frac{1}{2} \sum_{i j} \sum_{\alpha \beta} u_{i}^{\alpha} \Phi_{i j}^{\alpha \beta} u_{j}^{\beta}
$$

$M_{i}$ denotes the mass of an ion at site $i$. It equals $M_{\mathrm{A}}$ or $M_{\mathrm{B}}$. The dynamical matrix $\Phi_{i j}^{\alpha \beta}$ is also in general a random quantity.

\subsection{Electron Green's Function and Mass Operator}

In disordered systems where the distance between "impurities" is comparable to the interatomic distance of the host ${ }^{10}$ the coherence length 
(or Cooper pair size) is greatly reduced. The proper description of superconductivity in such systems requires the proper description of the Cooper pairs. The pairing in general takes place between time-reversed states and these cannot be represented as $\mathbf{k} \uparrow$ and $-\mathbf{k} \downarrow$ in disordered alloys, because $\mathbf{k}$ is not a good quantum number in these systems. Therefore, we have to start from the states in the site representation, describe the pairing (i.e., obtain an expression for the anomalous electron Green's function and mass operator), and only then average over various configurations in order to obtain quantities that can be compared with experiment.

To solve for the mass operator we use the equation of motion method for the two-time thermodynamic Green's function. ${ }^{36,37}$ The Green's function $\hat{G}_{i j}^{\alpha}(\omega)$ is a matrix in Nambu representation and is defined for a fixed configuration of ions in space by

$$
\hat{G}_{i j}^{\sigma}(\omega)=\left(\begin{array}{cc}
\left\langle a_{i \sigma} \mid a_{j \sigma}^{+}\right\rangle_{\omega}, & \left\langle\left\langle a_{i \sigma} \mid a_{j-\sigma}\right\rangle_{\omega}\right. \\
\left\langle\left\langle a_{i-\sigma}^{+} \mid a_{j \sigma}^{+}\right\rangle_{\omega},\right. & \left\langle\left\langle a_{i-\sigma}^{+} \mid a_{j \sigma}^{+}\right\rangle_{\omega}\right.
\end{array}\right)=\left\langle\left\langle\psi_{i \sigma} \mid \psi\right\rangle\right\rangle_{\omega}
$$

where $\psi_{j \sigma}^{+}=\left(a_{j \sigma}^{+}, a_{j-\sigma}\right)$ is the so-called Nambu field spinor. ${ }^{5}$ Differentiation of $\hat{G}_{i j}^{\sigma}\left(t-t^{\prime}\right)$ over the first time variable $t$ gives the following equation for the Green's function (6):

$$
\sum_{m} \hat{A}_{i m} \hat{G}_{m j}^{\sigma}(\omega)=\hat{I}_{i j}+U_{i} \hat{\tau}_{3} \hat{B}_{i j}+\sum_{m \beta} T_{i m}^{\beta} \hat{C}_{i m, j}
$$

where the caret denotes matrices in spin indices, $\hat{\tau}_{i}, i=1,2,3$, are the Pauli matrices, and

$$
\begin{gathered}
\hat{A}_{i m}=\omega \hat{\tau}_{0}-\left(\epsilon_{i} \delta_{i m}+t_{i m}\right) \hat{\tau}_{3} ; \quad \hat{I}_{i j}=\delta_{i j} \hat{\tau}_{0} \\
\hat{B}_{i j}=\left(\begin{array}{ll}
\left.\left\langle a_{i \sigma} n_{i-\sigma} \mid a_{j \sigma}^{+}\right\rangle\right\rangle_{\omega}, & \left.\left\langle a_{i \sigma} n_{i-\sigma} \mid a_{j-\sigma}\right\rangle\right\rangle_{\omega} \\
\left\langle\left\langle a_{i-\sigma}^{+} n_{i \sigma} \mid a_{j \sigma}^{+}\right\rangle_{\omega},\right. & \left\langle\left\langle a_{i-\sigma}^{+} n_{i \sigma} \mid a_{j-\sigma}\right\rangle_{\omega}\right.
\end{array}\right) \\
\hat{C}_{i m, j}=\left(\begin{array}{cc}
\left\langle u_{i m}^{\beta} a_{m \sigma} \mid a_{j \sigma}^{+}\right\rangle_{\omega}, & \left\langle\left\langle u_{i m}^{\beta} a_{m \sigma} \mid a_{j-\sigma}\right\rangle\right\rangle_{\omega} \\
\left\langle u_{m i}^{\beta} a_{m-\sigma}^{+} \mid a_{j \sigma}^{+}\right\rangle_{\omega}, & \left\langle u_{m i}^{\beta} a_{m-\sigma}^{+} \mid a_{j-\sigma}\right\rangle_{\omega}
\end{array}\right) \\
u_{i m}^{\alpha}=u_{i}^{\alpha}-u_{m}^{\alpha}
\end{gathered}
$$

To proceed we define the "irreducible" operator as in Refs. 29 and 33-35:

$$
\begin{aligned}
\left\langle{ }^{i r}\left(a_{i \sigma} n_{i-\sigma}\right) \mid a_{i \sigma}^{+}\right\rangle= & \left\langle\left\langle a_{i \sigma} n_{i-\sigma} \mid a_{j \sigma}^{+}\right\rangle\right\rangle_{\omega} \\
& -\left\langle n_{i-\sigma}\right\rangle\left\langle\left\langle a_{i \sigma} \mid a_{j \sigma}^{+}\right\rangle_{\omega}\right. \\
& +\left\langle a_{i \sigma} a_{i-\sigma}\right\rangle\left\langle\left\langle a_{i-\sigma}^{+} \mid a_{j \sigma}^{+}\right\rangle_{\omega}\right.
\end{aligned}
$$

which gives rise to new equations of the form (7) with $\hat{B}_{i m}$ replaced by 
${ }^{i r}\left(\hat{B}_{i m}\right)$ and $\hat{A_{i m}}$ replaced by

$$
\hat{A}_{1, i m}=\hat{A}_{i m}-U_{i}\left(\begin{array}{cc}
\left\langle n_{i-\sigma}\right\rangle, & -\left\langle a_{i \sigma} a_{i-\sigma}\right\rangle \\
-\left\langle a_{i-\sigma}^{+} a_{i \sigma}^{+}\right\rangle, & -\left\langle n_{i \sigma}\right\rangle
\end{array}\right) \delta_{i m}
$$

This means that we have extracted from the original Green's function the Hartree-Fock-Bogolubov ${ }^{3}$ mean field, given here by the difference $\left(\hat{A}_{1, i m}-\right.$ $\left.\hat{A}_{i m}\right)$. To proceed we write down the equations of motion for the Green's functions ${ }^{i r}\left(\hat{B}_{i j}\right)$ and $\hat{C}_{i m, j}$, differentiating them over the second time variable $t^{\prime}{ }^{29,35}$ Then we again go over to "irreducible" Green's functions, but now with respect to rhs operators (cf. Ref. 27). The set of equations obtained for the various Green's functions can be solved exactly. To this end we define the zeroth-order Green's functions as

$$
\sum_{m} \hat{A}_{1, i m} \hat{\mathscr{G}}_{m j}^{0 \sigma}(\omega)=I_{i j}
$$

and obtain the following exact equation:

$$
\hat{\mathscr{G}}_{i j}^{\sigma}(\omega)=\hat{\mathscr{G}}_{i j}^{0 \sigma}(\omega)+\sum_{n, m} \hat{\mathscr{G}}_{i n}^{0 \sigma}(\omega) \hat{K}_{n m}^{\sigma}(\omega) \hat{\mathscr{G}}_{m j}^{0 \sigma}(\omega)
$$

where the "scattering" operator $\hat{K}^{\sigma}$ is given by

$$
\begin{aligned}
& \hat{K}_{i l}^{\sigma}(\omega)=\sum_{m l^{\prime}} \hat{\tau}_{3}\left(\begin{array}{c}
\left\langle\left\langle^{i r}\left(\rho_{i m}^{-\sigma} a_{m \sigma}\right) \mid\left(\rho_{l l^{\prime}}^{-\sigma} a_{l^{\prime} \sigma}^{+}\right)^{i r}\right\rangle\right\rangle_{\omega}, \\
\left\langle{ }^{i r}\left(\rho_{i m}^{-\sigma} a_{m \sigma}\right) \mid\left(\rho_{l l^{\prime}}^{\sigma} a_{l-\sigma}\right)^{i r}\right\rangle_{\omega} \\
\\
\\
\left\langle\left\langle^{i r}\left(\rho_{i m}^{\sigma} a_{m-\sigma}^{+}\right) \mid\left(\rho_{l l^{\prime}}^{-\sigma} a_{l^{\prime} \sigma}^{+}\right)^{i r}\right\rangle_{\omega},\right. \\
\left\langle\left\langle^{i r}\left(\rho_{i m}^{\sigma} a_{m-\sigma}^{+}\right) \mid\left(\rho_{l l^{\prime}}^{\sigma} a_{l^{\prime}-\sigma}\right)^{i r}\right\rangle_{\omega}\right.
\end{array}\right) \hat{\tau}_{3} \\
& \rho_{i j}^{\sigma}=U_{i} n_{i o} \delta_{i j}+\sum_{\alpha} T_{i j}^{\alpha}\left(u_{i}^{\alpha}-u_{j}^{\alpha}\right)
\end{aligned}
$$

Equation (11) can be written in the form of the Dyson equation ${ }^{33,34}$

$$
\hat{\mathscr{G}}_{n j}^{\sigma}(\omega)=\hat{\mathscr{G}}_{n j}^{0 \sigma}(\omega)+\sum_{i l} \hat{\mathscr{G}}_{n i}^{\sigma \sigma}(\omega) \hat{\mathscr{M}}_{i l}^{\sigma}(\omega) \hat{\mathscr{G}}_{l j}^{\sigma}(\omega)
$$

where one introduces the mass operator $\hat{\mathcal{M}}_{i l}^{\sigma}(\omega)$, the "proper part" ${ }^{, 33}$ of the scattering operator $K_{i l}^{\sigma}(\omega)$. Denoting the random matrices in site space by $\hat{\mathscr{G}}, \hat{\mathscr{G}}^{0}$, and $\hat{\mathcal{M}}$, one can write the formal solution of (14) as

$$
\hat{\mathscr{G}}=\left[\left(\mathscr{G}^{0}\right)^{-1}-\mathscr{M}\right]^{-1}
$$

To find an expression for the mass operator $\hat{M}$ we proceed in the same way as done previously ${ }^{29,35}$ and express the Green's functions entering the operator $\hat{K}$ through the correlation function by means of the spectral 
theorem. ${ }^{36,37}$ We decouple these correlation functions in the following way:

$$
\begin{aligned}
\left\langle u_{m l}^{\beta}(t) a_{m \sigma}^{+}(t) u_{i n}^{\alpha} a_{n \sigma}\right\rangle & \approx\left\langle u_{m l}^{\beta}(t) u_{i n}^{\alpha}\right\rangle\left\langle a_{m \sigma}^{+}(t) a_{n \sigma}\right\rangle \\
\left\langle n_{l-\sigma}(t) a_{l \sigma}^{+}(t) n_{i-\sigma} a_{l \sigma}\right\rangle & \approx\left\langle n_{l-\sigma}(t) n_{i-\sigma}\right\rangle\left\langle a_{l \sigma}^{+}(t) a_{i \sigma}\right\rangle
\end{aligned}
$$

neglecting the vertex corrections according to the Midal-Eliashberg approach. ${ }^{4,6,29}$ Using again the spectral theorem on the rhs of (16), we obtain for the mass operator $\hat{M}$ (cf. Ref. [35])

$$
\hat{\mathscr{M}}_{i l}^{\sigma}(\omega)=\hat{\mathscr{M}}_{i l, \mathrm{ph}}^{e l-\omega}(\omega)+\hat{\mathscr{M}}_{i l, \sigma}^{c}(\omega)
$$

with the electron-phonon part given by

$$
\begin{aligned}
\hat{M}_{i l, \sigma}^{\mathrm{el-ph}}(\omega)= & \frac{1}{2} \iint_{-\infty}^{\infty} d \omega_{1} d \omega_{2} \frac{\operatorname{cth}\left(\beta \omega_{1} / 2\right)+\operatorname{th}\left(\beta \omega_{2} / 2\right)}{\omega-\omega_{1}-\omega_{2}} \\
& \times \sum_{m l^{\prime} \alpha \beta} \sum_{i m} T_{i m}^{\alpha}\left(-\frac{1}{\pi}\right) \operatorname{Im}\left\langle\left\langle u_{i m}^{\beta} \mid u_{l^{\prime}}^{\alpha}\right\rangle\right\rangle_{\omega_{1}+i \epsilon} \tau_{3}\left(-\frac{1}{\pi}\right) \\
& \times \operatorname{Im} \mathscr{G}_{m l^{\prime}}^{\sigma}\left(\omega_{2}+i \epsilon\right) \hat{\tau}_{3} T_{l^{\prime} l}^{\alpha}
\end{aligned}
$$

and the energy-dependent Coulomb part given by

$$
\begin{aligned}
\hat{\mathscr{M}}_{i l, \sigma}^{\mathrm{c}}(\omega)= & \frac{U_{i} U_{l}}{2 n^{2}} \iint_{-\infty}^{\infty} d \omega_{1} d \omega_{2} \frac{\operatorname{cth}\left(\beta \omega_{1} / 2\right)+\operatorname{th}\left(\beta \omega_{2} / 2\right)}{\omega-\omega_{1}-\omega_{2}} \\
& \times\left[\Gamma_{i l}^{\sigma, \sigma}\left(\omega_{1}\right) g_{i l}^{n}\left(\omega_{2}\right)-\Gamma_{i l}^{\sigma-\sigma}\left(\omega_{1}\right) g_{i l}^{s}\left(\omega_{2}\right)\right]
\end{aligned}
$$

with

$$
\begin{aligned}
& \Gamma_{i l}^{\sigma \sigma}(\omega)=\operatorname{Im}\left(\begin{array}{cc}
\left\langle\left\langle n_{i-\sigma} \mid n_{l-\sigma}\right\rangle\right\rangle_{\omega+i \epsilon}, & 0 \\
0, & \left\langle\left\langle n_{i \sigma} \mid n_{l \sigma}\right\rangle_{\omega+i \epsilon}\right.
\end{array}\right) \\
& g_{i l}^{n}(\omega)=\operatorname{Im}\left(\begin{array}{cc}
\left\langle\left\langle a_{i \sigma} \mid a_{l \sigma}^{+}\right\rangle_{\omega+i \epsilon},\right. & 0 \\
0, & \left\langle a_{i-\sigma}^{+} \mid a_{l \sigma}\right\rangle_{\omega+i \epsilon}
\end{array}\right), \\
& g_{i l}^{s}(\omega)=\operatorname{Im}\left(\begin{array}{cc}
0, & \left\langle a_{i \sigma} \mid a_{l-\sigma}\right\rangle_{\omega+i \epsilon} \\
\left\langle\left\langle a_{i-\sigma}^{+} \mid a_{l-\sigma}^{+}\right\rangle\right\rangle_{\omega+i \epsilon}, & 0
\end{array}\right)
\end{aligned}
$$

The elastic or Hartree-Fock-Bogolubov part of the Coulomb mass operator, not included in (17) and (19), can be written as [cf. its definition in (9)]

$$
\mathcal{M}_{i l \sigma}^{\mathrm{HF}}(\omega)=\frac{U_{i}}{2} \delta_{i} \hat{\tau}_{3}-\frac{U_{i}}{2} \int_{-\infty}^{\infty} d \omega_{1} \operatorname{th} \frac{\beta \omega_{1}}{2} \hat{\tau}_{3}\left[-\frac{1}{\pi} \operatorname{Im} \hat{\mathscr{G}}_{i i}^{-\sigma}\left(\omega_{1}+i \epsilon\right)\right] \hat{\tau}_{3} \delta_{i l}
$$


The equations (14) and (17)-(20) form a set of a self-consistent equations for the determination of the random Green's function and mass operator. The calculation of the phonon Green's function entering the electronphonon part of the mass operator is discussed in the following section.

\subsection{The "Renormalized" Phonon Green's Function}

The general scheme of the calculation is the same as for the electron Green's function. The phonon Green's function is defined as ${ }^{35}$

$$
D_{i j}^{\alpha \beta}\left(t-t^{\prime}\right)=\left\langle\left\langle u_{i}^{\alpha}(t) \mid u_{j}^{\beta}\left(t^{\prime}\right)\right\rangle\right\rangle=-i \theta\left(t-t^{\prime}\right)\left\langle\left[u_{i}^{\alpha}(t), u_{j}^{\beta}\left(t^{\prime}\right)\right]\right\rangle
$$

We differentiate it twice over the time $t$ and then twice over the time $t^{\prime}$. The zeroth-order Green's function is defined as

$$
\sum_{n \gamma}\left[M_{i} \omega^{2} \delta_{i n} \delta_{\alpha \gamma}-\Phi_{i n}^{\alpha \gamma}\right] D_{n j}^{0 \gamma \beta}(\omega)=\delta_{i j} \delta_{\alpha \beta}
$$

and enables us to write down the Dyson equation $D=D^{0}+D^{0} \Pi D$, with the phonon mass operator $\Pi$ (polarization operator) given by

$$
\begin{aligned}
\Pi_{m m^{\prime}}^{\gamma \gamma^{\prime}}(\omega)= & \frac{1}{2 \pi^{2}} \iint_{-\infty}^{\infty} d \omega_{1} d \omega_{2} \frac{\operatorname{th}\left(\beta \omega_{1} / 2\right)-\operatorname{th}\left(\beta \omega_{2} / 2\right)}{\omega-\omega_{1}+\omega_{2}} \\
& \times \sum_{n l} \sum_{n^{\prime} l^{\prime}} \sum_{\sigma}\left\{\left(\delta_{m n}-\delta_{m l}\right)\right. \\
& \times T_{n l}^{\gamma}\left[\operatorname { I m } \langle \langle a _ { l \sigma } | a _ { n ^ { \prime } \sigma } ^ { + } \rangle \rangle _ { \omega _ { 1 } + i \epsilon } \operatorname { I m } \left\langle\left\langle a_{l^{\prime} \sigma} \mid a_{n \sigma}^{+}\right\rangle_{\omega_{2}-i \epsilon^{\prime}}\right.\right. \\
& \left.-\operatorname{Im}\left\langle\left\langle a_{n-\sigma}^{+} \mid \dot{a}_{n^{\prime} \sigma}\right\rangle\right\rangle_{\omega_{1}+i \epsilon} \operatorname{Im}\left\langle\left\langle a_{l^{\prime} \sigma} \mid a_{l \sigma}\right\rangle_{\omega_{2}+i \epsilon}\right] T_{n^{\prime} l^{\prime}}^{\gamma^{\prime}}\left(\delta_{n^{\prime} m^{\prime}}-\delta_{l^{\prime} m^{\prime}}\right)\right\}
\end{aligned}
$$

Note that the phonon spectrum in the superconducting state is additionally renormalized as compared to the normal state. ${ }^{27,32,38}$ To obtain the formula (23) for the polarization operator $\Pi$ we have neglected the vertex corrections, as in (16).

\section{CONFIGURATIONAL AVERAGING}

In this section we discuss different attempts at averaging. Our main task is to obtain the averaged system of equations describing the superconducting alloy. For a given, fixed configuration of atoms in a lattice, these are given by the set of equations (14) and (17)-(20). Roughly speaking, we need the configurationally averaged Green's Function $\langle\hat{\mathscr{G}}(z)\rangle=\bar{G}(z)$ and total mass operator $\left\langle\mathcal{M}^{\text {tot }}(z)\right\rangle=\bar{M}^{\text {tot }}(z)$, where

$$
\hat{\mathscr{M}}^{\mathrm{tot}}(z)=\hat{\mathscr{M}}^{\mathrm{HF}}+\hat{\mathscr{M}}^{\mathrm{el-ph}}(z)+\hat{\mathscr{M}}^{\mathrm{c}}(z)
$$


For later convenience we rewrite Eq. (14) as

$$
\sum_{l}\left[z \hat{\tau}_{0} \delta_{i l}-\epsilon_{i} \hat{\tau}_{3} \delta_{i l}-t_{i l} \hat{\tau}_{3}-\hat{\mathscr{M}}_{i l, \sigma}^{\mathrm{tot}}(z)\right] \mathscr{G}_{l j}^{\sigma}(z)=\delta_{i j}
$$

In this paper we are not interested in the dynamical effect of the electronelectron interaction and neglect the mass operator $\hat{\mathcal{M}}_{i l}^{\mathrm{c}}(z)$. Thus the electron correlations are treated in the Hartree-Fock approximation.

We start the discussion of averaging with the simplest possibility, where only the random energy levels $\epsilon_{i}$ are described in the CPA and the other random parameters $U_{i}, T_{i j}$ are averaged to lowest order in the concentration $x$.

\subsection{The Simplest Method of Averaging}

In the following we assume the hopping integrals $t_{i j}$ to be nonrandom, periodic quantities, or replace the actual parameters by their averaged values, i.e.,

$$
t_{i j} \rightarrow \bar{t}_{i j}=x^{2} t_{i j}^{\mathrm{AA}}+2 x(1-x) t_{i j}^{\mathrm{AB}}+(1-x)^{2} t_{i j}^{\mathrm{BB}}
$$

The average of the alloy Green's function $\hat{\mathscr{G}}=\hat{\mathscr{G}}^{0}+\hat{\mathscr{G}}^{0} \hat{\mathscr{M}}^{1} \hat{\mathscr{G}}$, where $\hat{\mathscr{M}}^{1}=$ $\hat{\mathscr{M}}^{\mathrm{HF}}+\hat{\mathscr{M}}^{\mathrm{el-ph}}$ and $\hat{\mathscr{G}}^{0}$ is defined by

$$
\sum_{l}\left(z \hat{\tau}_{0} \delta_{i l}-\epsilon_{i} \hat{\tau}_{3} \delta_{i l}-\bar{t}_{i l} \hat{\tau}_{3}\right) \hat{G}_{l j}^{0}(z)=\delta_{i j}
$$

is assumed to be

$$
\hat{\bar{G}}=\hat{\bar{G}}_{\mathrm{CPA}}^{0}+\hat{\bar{G}}_{\mathrm{CPA}}^{0}\left\langle\hat{M}^{1}\right\rangle \hat{\bar{G}}
$$

$\bar{G}_{\mathrm{CPA}}^{0}$ denotes the CPA averaged Green's function defined in Eq. (27). In order to obtain the lowest order estimation to $\left\langle\hat{M}^{1}\right\rangle$ we replace the Green's functions entering the definition of $\hat{M}^{1}$ by their averaged values while the remaining random single-site parameters, $\alpha_{i}=U_{i}, q_{0}^{i}$, etc., or their product average in the following manner

$$
\left\langle\alpha_{i} \alpha_{j}\right\rangle= \begin{cases}\left\langle\alpha_{i}^{2}\right\rangle=x \alpha_{\mathrm{A}}^{2}+y \alpha_{\mathrm{B}}^{2} ; & i=j \\ \left\langle\alpha_{i}\right\rangle\left\langle\alpha_{j}\right\rangle=\left(x \alpha_{\mathrm{A}}+y \alpha_{\mathrm{B}}\right)^{2} ; & i \neq j\end{cases}
$$

The above averaging scheme is rather crude but workable. It gives some insight into the problem, and moreover enables us to derive the nonlinearized Eliashberg equations of superconductivity in alloys. In a sense this scheme resembles the so-called Anderson limit of constant order parameter studied in the CPA by various authors. ${ }^{14,20}$ 
Fourier-transforming the averaged equation (28) and expressing the averaged mass operator $\bar{M}_{\mathbf{k}}^{1}(\omega)$ in terms of the Pauli matrices $\hat{\tau}_{i}$ in a standard way, ${ }^{4-8,12}$

$$
\hat{M}_{\mathbf{k}}^{1}(\omega)=\left[1-Z_{\mathbf{k}} \dot{(\omega)}\right] \omega \hat{\tau}_{0}+\chi_{\mathbf{k}}(\omega) \hat{\tau}_{3}+\varphi_{\mathbf{k}}(\omega) \hat{\tau}_{1}
$$

we arrive at the equations

$$
\begin{aligned}
{[1-Z(\omega)] \omega=} & -\int_{-\infty}^{\infty} d \omega^{\prime} K\left(\omega^{\prime}, \omega\right) \operatorname{Re} \frac{\omega^{\prime}}{\left[\left(\omega^{\prime}\right)^{2}-\Delta^{2}\left(\omega^{\prime}\right)\right]^{1 / 2}} \operatorname{sign} \omega^{\prime} \\
\Delta(\omega) Z(\omega)= & \int_{-\infty}^{\infty} d \omega^{\prime} K\left(\omega^{\prime}, \omega\right) \operatorname{Re} \frac{\Delta\left(\omega^{\prime}\right)}{\left[\left(\omega^{\prime}\right)^{2}-\Delta^{2}\left(\omega^{\prime}\right)\right]^{1 / 2}} \operatorname{sign} \omega^{\prime} \\
& -V_{\mathrm{C}} \int_{0}^{\omega_{\mathrm{C}}} d \omega^{\prime} \text { th } \frac{\beta \omega^{\prime}}{2} \operatorname{Re} \frac{\Delta\left(\omega^{\prime}\right)}{\left[\left(\omega^{\prime}\right)^{2}-\Delta^{2}\left(\omega^{\prime}\right)\right]^{1 / 2}}
\end{aligned}
$$

where $\Delta(\omega)=\varphi(\omega) / Z(\omega)$ and

$$
V_{\mathrm{C}}=N\left(\epsilon_{\mathrm{F}}\right)\left\langle U_{i}\right\rangle /\left[1+N\left(\epsilon_{\mathrm{F}}\right)\left\langle U_{i}\right\rangle \ln \frac{\epsilon_{\mathrm{F}}}{\omega_{\mathrm{C}}}\right]
$$

$N\left(\epsilon_{\mathrm{F}}\right)$ is the density of states of an alloy at fermi energy $\epsilon_{\mathrm{F}}$, and the kernel $K\left(\omega^{\prime}, \omega\right)$ is expressed in the usual way ${ }^{12}$ through the electron-phonon spectral (or Eliashberg) function $\alpha^{2}(z) F(z)$ containing all the essential information about the system:

$$
\begin{aligned}
& \alpha^{2}(z) F(z) \\
&=\int_{S_{\mathrm{F}}} \frac{d^{2} \mathbf{k}}{v_{\mathbf{k}}} \int_{S_{\mathrm{F}}} \frac{d^{2} \mathbf{q}}{v_{\mathbf{q}}} \sum_{\lambda}\left\{\left|g_{1}^{\lambda}(\mathbf{k}, \mathbf{q})\right|^{2}\left[-\frac{1}{\pi} \operatorname{Im} \bar{D}_{\mathbf{k}-\mathbf{q}, \lambda}(z+i \epsilon)\right]\right. \\
&\left.+\int_{S_{\mathbf{F}}} \frac{d^{2} \mathbf{p}_{\mathbf{p}}}{v_{\mathbf{p}}}\left|g_{2}^{\lambda}(\mathbf{k}, \mathbf{q}, \mathbf{p})\right|^{2}\left[-\frac{1}{\pi} \operatorname{Im} \bar{D}_{\mathbf{k}-\mathbf{p}-\mathbf{q}}(z+i \epsilon)\right]\right\} / \int_{S_{\mathbf{F}}} \frac{d^{2} \mathbf{k}}{v_{\mathbf{k}}} \\
&\left|g_{1}^{\lambda}(\mathbf{k}, \mathbf{q})\right|^{2}=\frac{Q_{2}}{2 M_{\mathrm{A}} d^{2}} \sum_{\alpha}\left[e_{\mathbf{k}-\mathbf{q}, \lambda}^{\alpha}\left(v_{\mathbf{k}}^{\alpha}-v_{\mathbf{q}}^{\alpha}\right)\right]^{2} \\
&\left|g_{1}^{\lambda}(\mathbf{k}, \mathbf{q}, \mathbf{p})\right|^{2}=\frac{Q_{1}-Q_{2}}{8 M_{\mathrm{A}} d^{2}} \sum_{\alpha}\left[e_{\mathbf{k}-\mathbf{q}-\mathbf{p}, \lambda}^{\alpha}\left(v_{\mathbf{k}}^{\alpha}-v_{\mathbf{p}+\mathbf{q}}^{\alpha}+v_{\mathbf{k}-\mathbf{p}}^{\alpha}-v_{\mathbf{q}}^{\alpha}\right)\right]^{2} \\
& Q_{1}=x q_{\mathrm{A}}^{2}+y q_{\mathrm{B}}^{2} ; \quad Q_{2}=x^{2} q_{\mathrm{A}}^{2}+2 x y q_{\mathrm{A}} q_{\mathrm{B}}+y^{2} q_{\mathrm{B}}^{2} \\
& \epsilon_{\mathbf{k}}=N^{-1} \sum_{i j} \overline{t_{i j}} \exp \left[i \mathbf{k}\left(\mathbf{R}_{i}-\mathbf{R}_{i}\right)\right], \quad v_{\mathbf{k}}^{\alpha}=\frac{\partial \epsilon_{\mathbf{k}}}{\partial k^{\alpha}}
\end{aligned}
$$

$M_{\mathrm{A}}$ is the mass of an A-type atom, $d$ denotes the distance between nearest neighbors in a lattice, and $\mathbf{e}_{\mathbf{k}, \lambda}$ and $\bar{D}_{\mathbf{k}, \lambda}(z)$ denote, respectively, the phonon polarization vector and the averaged Green's function of phonon branch $\lambda$. 
The phonon Green's function $\bar{D}_{k \lambda}$ itself is a solution of the equation (shorthand notation is used)

$$
\bar{D}=\bar{D}^{0}+\bar{D}^{\circ} \bar{\Pi} \bar{D}
$$

where $\bar{D}^{0}$ as defined in (22) is calculated in the CPA, but the phonon mass operator $\bar{\Pi}(\omega)$ giving the renormalization of the phonon spectrum in an alloy is calculated here in a similar way as $\bar{M}$.

In general it is important to use the fully renormalised phonon Green's function because the anomalous phonon contribution for high $T_{c}$ comes mainly ${ }^{32}$ from the phonon linewidth $[\sim \operatorname{Im} \bar{\Pi}(\omega+i \epsilon)]$ and the renormalization can remarkably change the spectrum of the superconductor, giving rise to a new localized phonon mode, as recently discussed by Machida. ${ }^{38}$

\subsection{General Averaging Scheme}

All the quantities of the theory developed in Section 2, such as mass operators $\hat{\mathscr{M}}_{i l, \sigma}(\omega), \hat{\Pi}_{i l}(\omega)$, Green's functions, etc., depend on the configuration of the whole alloy. Most important, however, is the dependence on the occupancy of the so-called terminal points $i, l$. The rest of the atoms can be replaced by an effective medium. This means that we replace the functions $\hat{M}_{i l, \sigma}(\omega), \hat{\mathscr{G}}_{i l}^{\sigma}(\omega)$, etc., by their conditionally averaged counterparts

$$
\hat{M}_{i l, \sigma}(\omega)=\left\langle\hat{M}_{i l, \sigma}(\omega)\right\rangle_{i l}^{\{j\}} ; \quad \hat{G}_{i l}^{\sigma}(\omega)=\left\langle\hat{\mathscr{G}}_{i l}^{\sigma}(\omega)\right\rangle_{i l}^{\{j\}} ; \ldots
$$

where $\langle\cdots\rangle_{i l}^{\{j\}}$ denotes the configurational conditional averaging over all lattice sites $\{j\}$ different from $i$ and $l$, the conditions being the fixed types of atoms at sites $i$ and $l$. Evaluation of various conditional averages $M_{i l}^{\mathrm{AA}}, M_{i l}^{\mathrm{AB}}, \ldots$ requires in turn knowledge of the conditionally averaged electron and phonon Green's functions. The best way to calculate them is to use the off-diagonal CPA developed by Blackman, et al. ${ }^{39}$ for electrons and its extension to phonons. ${ }^{40}$ The resulting set of equations is difficult to solve numerically and therefore we will not discuss it further.

To make the problem tractable we resort in the next subsection to additional approximations leading to the single-site description.

\subsection{The Random Contact Model}

In the contact model, electron scattering processes caused by the electron-electron and electron-phonon interactions are taken into account only if the two electrons are initially both at the same site $i$ and finally both at another site $j .{ }^{24-26}$ In our tight binding approach this means that we neglect all off-diagonal (in site indices) matrix elements of the electron 
and phonon Green's functions and of the mass operator. Thus we obtain

$$
\begin{aligned}
\hat{\mathcal{M}}_{i i}^{\sigma}(\omega)= & \epsilon_{i} \hat{\tau}_{3}-\frac{U_{i}}{2} \int_{-\infty}^{\infty} d \omega_{2} \operatorname{th} \frac{\beta \omega_{2}}{2} \hat{\tau}_{3}\left\{-\frac{1}{\pi} \operatorname{Im} \hat{\mathscr{G}}_{i i}^{-\sigma}\left(\omega_{2}+i \epsilon\right)\right\} \hat{\tau}_{3} \\
& -\frac{1}{2} \iint_{-\infty}^{\infty} d \omega_{1} d \omega_{2} \frac{\operatorname{cth}\left(\beta \omega_{1} / 2\right)+\operatorname{th}\left(\beta \omega_{2} / 2\right)}{\omega-\omega_{1}-\omega_{2}} \\
& \times \sum_{m \alpha} T_{i m}^{\alpha}\left\{-\frac{1}{\pi} \operatorname{Im}\left[D_{i i}^{\alpha}\left(\omega_{1}+i \epsilon\right)\right.\right. \\
& \left.\left.+D_{m m}^{\alpha}\left(\omega_{1}+i \epsilon\right)\right]\right\} \hat{\tau}_{3}\left\{-\frac{1}{\pi} \operatorname{Im} \mathscr{G}_{m m}^{\sigma}\left(\omega_{2}+i \epsilon\right)\right\} \hat{\tau}_{3} T_{m i}^{\alpha}
\end{aligned}
$$

Note that we have incorporated the random energy levels $\epsilon_{i}$ into the definition of the total mass operator matrix. Because of the definition of the electron-phonon interaction parameters $T_{i m}^{\alpha}$ [cf. their definition in Eq. (4)] the sum over $m$ in Eq. (38) is limited to nearest neighbor sites to $i$. This sum gives rise to some sort of configurational averaging. The configurational average of any function $\mathscr{G}$ in the site representation can be defined as

$$
G_{i j}(\omega)=\frac{1}{N} \sum_{l} \mathscr{G}_{i+l, j+l}
$$

where the subscript $l$ goes over all $N$ randomly occupied sites in a sample. Noting this fact and denoting the distance between neighboring sites as previously by $d=\left|\mathbf{R}_{m}-\mathbf{R}_{i}\right|$ and $d_{\alpha}=R_{m}^{\alpha}-R_{i}^{\alpha}$, we can rewrite Eq. (38) for an atom of type $\mathrm{A}$ at site $i$ as

$$
\begin{aligned}
\hat{M}_{\mathrm{A}}^{\sigma}(\omega)= & \epsilon_{\mathrm{A}} \hat{\tau}_{3}-\frac{U_{\mathrm{A}}}{2} \int_{-\infty}^{\infty} \mathrm{d} \omega_{2} \operatorname{th} \frac{\beta \omega_{2}}{2} \hat{\tau}_{3}\left\{-\frac{1}{\pi} \operatorname{Im} \hat{G}_{\mathrm{A}}^{-\sigma}\left(\omega_{2}+i \epsilon\right)\right\} \hat{\tau}_{3} \\
& -\frac{1}{2 \pi^{2}} \iint_{-\infty}^{\infty} d \omega_{1} d \omega_{2} \frac{\operatorname{cth}\left(\beta \omega_{1} / 2\right)+\operatorname{th}\left(\beta \omega_{2} / 2\right)}{\omega-\omega_{1}-\omega_{2}} \\
& \times \sum_{\alpha} \frac{\vec{t}^{2} d_{\alpha}^{2}}{d^{2}}\left\{2 x q_{\mathrm{A}}^{2} \operatorname{Im} D_{\mathrm{A}}^{\alpha}\left(\omega_{1}+i \epsilon\right)\right. \\
& \times \hat{\tau}_{3} \operatorname{Im} \hat{G}_{\mathrm{A}}^{\sigma}\left(\omega_{2}+i \epsilon\right) \hat{\tau}_{3}+\frac{1}{4}\left(q_{\mathrm{A}}+q_{\mathrm{B}}\right)^{2} y\left[\operatorname{Im} D_{\mathrm{A}}^{\alpha}\left(\omega_{1}+i \epsilon\right)\right. \\
+ & \left.\left.\operatorname{Im} D_{\mathrm{B}}^{\alpha}\left(\omega_{1}+i \epsilon\right)\right] x \hat{\tau}_{3} \operatorname{Im} \hat{G}_{\mathrm{B}}^{\sigma}\left(\omega_{2}+i \epsilon\right) \hat{\tau}_{3}\right\}
\end{aligned}
$$

with a similar formula for $M_{\mathrm{B}}^{\sigma}(\omega)$. Here $\bar{t}$ denotes the value of the hopping integral for neighboring atoms in a cubic lattice [cf. Eq. (26)]. According 
to the discussion in Section 3.2 and in order to have a true single-site description of $\hat{M}$, we have conditionally averaged Eq. (38) with the condition $i=\mathrm{A}$. Here $G_{\mathrm{A}}\left(D_{\mathrm{A}}\right)$ denotes the conditionally averaged electron (phonon) Green's function. The third term in (39) results from the electron-phonon interaction and has exactly the same form as Lustfeld's ${ }^{26}$ expression for $\sigma_{1, \mathrm{~A}}$ [his Eq. (8)].

The above single-site matrices $\hat{M}_{i}^{\sigma}(\omega), i=\mathrm{A}, \mathrm{B}$, are the only random quantities in our model and serve as input parameters in the matrix CPA equations. The outputs of these equations are: (i) the coherent potential matrix $\hat{\Sigma}^{\sigma}(\omega)$ replacing $M_{i}^{\sigma}(\omega)$ in an effective medium and (ii) the Green's function $\bar{G}^{\sigma}(\omega)$ describing the properties of the averaged system. As usual, the existence of a nonzero solution for the part of the $\hat{\Sigma}^{\sigma}(\omega)$ matrix that is off-diagonal in the spin indices (i.e., the anomalous part) determines the superconducting transition temperature $T_{\mathrm{c}}$.

The model, as stated above, is appropriate for discussing the possible coexistence between superconductivity and magnetism, but this is outside the scope of the present paper. Therefore in the following we omit the spin index $\sigma$.

\subsection{CPA Equations for Superconductivity in the Contact Model}

Here we briefly discuss the calculation of the averaged electron Green's functions $\hat{\bar{G}}(z)$ and $\hat{G}_{i}(z), i=\mathrm{A}, \mathrm{B}$. The averaged Green's function $\overline{\hat{G}}(z)$ is related to the configuration-dependent one $\hat{\mathscr{G}}(z)$ by $^{41}$

$$
\hat{\mathscr{G}}(z)=\hat{\bar{G}}(z)+\hat{\bar{G}}(z) \hat{\tau}(z) \hat{\bar{G}}(z)
$$

where the scattering operator $\hat{\tau}$ refers to the whole system. In the single-site CPA the condition $\langle\hat{\tau}\rangle=0$ determining the averaged Green's function is replaced by the following ${ }^{41}$ :

$$
\left\langle\hat{T}_{i}\right\rangle=x \hat{T}_{\mathrm{A}}+y \hat{T}_{\mathrm{B}}=0 ; \quad \hat{T}_{i}=\langle\tau\rangle_{i}^{\{j\}}
$$

with the single-site $T$-matrix

$$
\hat{T}_{i}=\hat{V}_{i}+\hat{T}_{i} \hat{V}_{i} \hat{\bar{G}}
$$

Here

$$
\begin{gathered}
\hat{V}_{i}=\left(\begin{array}{cc}
M_{11}^{i}(z)-\Sigma_{11}(z), & M_{12}^{i}(z)-\Sigma_{12}(z) \\
M_{12}^{* i}\left(z^{*}\right)-\Sigma_{12}^{*}\left(z^{*}\right), & -M_{11}^{i}(-z)+\Sigma_{11}(z)
\end{array}\right) \\
\hat{\bar{G}}(z)=\left(\begin{array}{cc}
\bar{G}_{11}(z), & \bar{G}_{12}(z) \\
\bar{G}_{12}^{*}\left(z^{*}\right), & -\bar{G}_{11}(-z)
\end{array}\right)
\end{gathered}
$$


and

$$
\begin{aligned}
& \bar{G}_{11}(z)=\frac{1}{N} \sum_{\mathbf{k}} \frac{z+\epsilon_{\mathbf{k}}+\Sigma_{11}(-z)}{\left[z-\epsilon_{\mathbf{k}}-\Sigma_{11}(z)\right]\left[z+\epsilon_{\mathbf{k}}+\Sigma_{11}(-z)\right]-\Sigma_{12}(z) \Sigma_{12}^{*}\left(z^{*}\right)} \\
& \bar{G}_{12}(z)=\frac{1}{N} \sum_{\mathbf{k}} \frac{\Sigma_{12}(z)}{\left[z-\epsilon_{\mathbf{k}}-\Sigma_{12}(z)\right]\left[z+\epsilon_{\mathbf{k}}+\Sigma_{11}(-z)\right]-\Sigma_{12}(z) \Sigma_{12}^{*}\left(z^{*}\right)}
\end{aligned}
$$

A very important relation connecting the anomalous and normal parts of (44) follows from the last two equations, namely

$$
\bar{G}_{12}(z)=\frac{\bar{G}_{11}(z)-\bar{G}_{11}(-z)}{2 z+\Sigma_{11}(-z)-\Sigma_{11}(z)} \Sigma_{12}(z)
$$

To close the set of equations (38) and (40)-(46) we need the expression for the single-site Green's function $\hat{G}_{i}(z)$. In the CPA it is given by ${ }^{41}$

$$
\hat{G}_{i}(z)=\hat{\bar{G}}(z)+\hat{\bar{G}}(z) \hat{T}_{i}(z) \hat{\bar{G}}(z)=\left[1-\hat{\bar{G}}\left(\hat{M}_{i}-\Sigma\right)\right]^{-1} \hat{\bar{G}}, \quad i=\mathrm{A}, \mathrm{B}
$$

The resulting set can be solved numerically and the transition temperature $T_{c}$ determined. At this temperature there is a nonzero solution for the anomalous part of these equations. Therefore we expect that at $T \rightarrow T_{c}$, $\Sigma_{12}(z) \rightarrow 0$ and $M_{12}^{i}(z) \rightarrow 0$, thus making possible linearization and simplification of the problem. This is the subject of the next section.

\subsection{Linearized Equations and the Transition Temperature}

The simplest way to linearize the equations of the previous sections with respect to $\Sigma_{12}(z)$ and $M_{12}^{i}(z)$ is to write every matrix $\hat{F}$ as a sum of normal $\hat{F}^{\mathrm{n}}$ (diagonal) and anomalous (i.e., purely off-diagonal, superconducting) $\hat{F}^{\mathrm{s}}$ parts and use the matrix identity $(A-B)^{-1}=$ $A^{-1}+A^{-1} B(A-B)^{-1}$ repeatedly. Up to linear order in $\Sigma_{12}$ the diagonal part of (41) gives the so-called Soven equation, ${ }^{42}$

$$
\begin{aligned}
\Sigma_{11}(z)= & x M_{11}^{\mathrm{A}}(z)+y M_{11}^{\mathrm{B}}(z) \\
& -\left[M_{11}^{\mathrm{A}}(z)-\Sigma_{11}(z)\right] \bar{G}_{11}(z)\left[M_{11}^{\mathrm{B}}(z)-\Sigma_{11}(x)\right] \\
\bar{G}_{11}(z)= & \frac{1}{N} \sum_{\mathbf{k}} \frac{1}{z-\epsilon_{\mathbf{k}}-\Sigma_{11}(z)}
\end{aligned}
$$

while the off-diagonal part can be written as

$$
\bar{G}_{12}(z)=\left\langle G_{11}^{i}(z)\left[\frac{\bar{G}_{12}(z)}{\bar{G}_{11}(z) \bar{G}_{11}(-z)}+\Sigma_{12}(z)-M_{12}^{i}(z)\right] G_{11}^{i}(-z)\right\rangle
$$


Noting the definition $G_{11}^{i}(z)=\left[G_{11}^{-1}(z)-M_{11}^{i}(z)+\Sigma_{11}(z)\right]^{-1}$ and the identity

$$
\begin{aligned}
G_{11}^{i}(z)-G_{11}^{i}(-z)= & G_{11}^{i}(z)\left[\frac{\bar{G}_{11}(-z)-\bar{G}_{11}(z)}{\bar{G}_{11}(z) \bar{G}_{11}(-z)}+\Sigma_{11}(z)-\Sigma_{11}(-z)\right. \\
& \left.+M_{11}^{i}(-z)-M_{11}^{i}(z)\right] G_{11}^{i}(-z)
\end{aligned}
$$

and defining the auxiliary function $\tilde{\Sigma}_{12}(z),{ }^{17,21,25}$

$$
\tilde{\Sigma}_{12}(z)=\Sigma_{12}(z) \frac{2 z}{2 z+\Sigma_{11}(-z)-\Sigma_{11}(z)}
$$

we obtain from (49) and (46) the CPA equation for $\tilde{\Sigma}_{12}(z)$ :

$$
\begin{aligned}
& \left\langle G_{12}^{i}(z) \tilde{\Sigma}_{12}(z) \frac{2 z-M_{11}^{i}(-z)+M_{11}^{i}(z)}{2 z} G_{11}^{i}(-z)\right\rangle \\
& \quad=\left\langle G_{11}^{i}(z) M_{12}^{i}(z) G_{11}^{i}(-z)\right\rangle
\end{aligned}
$$

Note that Eq. (51) has the structure of the so-called Abrikosov-Gorkov relation. ${ }^{10,21,26}$ It expresses the additional changes of $\Sigma_{12}(z)$ due to the disorder in the normal part of the problem. It is easy to verify that

$$
\begin{aligned}
G_{12}^{i}(z)= & \left\{G_{11}^{i}(z)-G_{11}^{i}(-z)-G_{11}^{i}(z)\left[2 z+M_{11}^{i}(z)-M_{11}^{i}(-z)\right] G_{11}^{i}(-z)\right\} \\
& \times \tilde{\Sigma}_{12}(z) / 2 z+G_{11}^{i}(z) M_{12}^{i}(z) G_{11}^{i}(z)
\end{aligned}
$$

Equation (38) or (29) and (53) determine the input parameters $M_{12}^{i}(z)$ for (52). It is worthwhile to note the presence of the terms $\left[M^{i}(-z)-M^{i}(z)\right]$ in (52) and (53). They express an additional influence of the electronphonon disorder (only the electron-phonon part of $\hat{M}_{i}$ is energy dependent in our treatment) on the superconducting behavior of an alloy. However, we expect this effect to be weak and neglect it. ${ }^{25,26}$

Combining Eqs. (50), (53), and (38) we obtain from (52) the equation

$$
\begin{aligned}
\tilde{\Sigma}_{12}(\omega)= & \int_{-\infty}^{\infty} d \omega^{\prime} K_{\text {eff }}\left(\omega^{\prime}, \omega\right) \operatorname{Re} \frac{\tilde{\Sigma}_{12}\left(\omega^{\prime}+i \epsilon\right)}{\omega^{\prime}} \\
& -N\left(\epsilon_{\mathrm{F}}\right) U_{\text {eff }} \int_{0}^{\infty} d \omega^{\prime} \text { th } \frac{\beta \omega^{\prime}}{2} \operatorname{Re} \frac{\tilde{\Sigma}_{12}\left(\omega^{\prime}+i \epsilon\right)}{\omega^{\prime}}
\end{aligned}
$$

replacing the Eliashberg equation for the order parameter $\Delta(\omega)$ [cf. Eq. (32)]. The kernel $K_{\text {eff }}$ is defined as usual

$$
K_{\mathrm{eff}}\left(\omega^{\prime}, \omega\right)=\int_{-\infty}^{\infty} \frac{}{d z \alpha^{2}(z) F(z)} \frac{\operatorname{cth}(\beta z / 2)+\operatorname{th}\left(\beta \omega^{\prime} / 2\right)}{z+\omega^{\prime}-\omega-i \epsilon}
$$


where the Eliashberg function is given by

$$
\begin{aligned}
\overline{\alpha^{2}(\omega) F(\omega)}= & 2 \sum_{\alpha} \vec{t}^{2} \frac{d_{\alpha}^{2}}{d^{2}}\left\{x^{2} q_{\mathrm{A}}^{2} N_{\mathrm{A}}^{2}\left(\epsilon_{\mathrm{F}}\right)\left[-\frac{1}{\pi} \operatorname{Im} D_{\mathrm{A}}^{\alpha}(\omega+i \epsilon)\right]\right. \\
& +\frac{1}{4} x y\left(q_{\mathrm{A}}+q_{\mathrm{B}}\right)^{2} N_{\mathrm{A}}\left(\epsilon_{\mathrm{F}}\right) N_{\mathrm{B}}\left(\epsilon_{\mathrm{F}}\right) \\
& \times\left[-\frac{1}{\pi} \operatorname{Im} D_{A}^{\alpha}(\omega+i \epsilon)-\frac{1}{\pi} \operatorname{Im} D_{\mathrm{B}}^{\alpha}(\omega+i \epsilon)\right] \\
& \left.+y^{2} q_{\mathrm{B}}^{2} N_{\mathrm{B}}^{2}\left(\epsilon_{\mathrm{F}}\right)\left[-\frac{1}{\pi} \operatorname{Im} D_{\mathrm{B}}^{\alpha}(\omega+i \epsilon)\right]\right\} \frac{1}{N\left(\epsilon_{\mathrm{F}}\right)} \\
& N\left(\epsilon_{\mathrm{F}}\right) U_{\mathrm{eff}}=\left\langle U_{i} N_{i}^{2}\left(\epsilon_{\mathrm{F}}\right) / N\left(\epsilon_{\mathrm{F}}\right)\right\rangle
\end{aligned}
$$

$N_{i}\left(\epsilon_{\mathrm{F}}\right)$ and $N\left(\epsilon_{\mathrm{F}}\right)$ denote, respectively, the partially and totally averaged electron densities of states at the Fermi level

$$
\begin{aligned}
& N_{i}\left(\epsilon_{\mathrm{F}}\right)=-\frac{1}{\pi} \operatorname{Im} G_{11}^{i}\left(\epsilon_{\mathrm{F}}\right), \quad i=\mathrm{A}, \mathrm{B} \\
& N\left(\epsilon_{\mathrm{F}}\right)=-\frac{1}{\pi} \operatorname{Im} \bar{G}_{11}\left(\epsilon_{\mathrm{F}}\right)=x N_{\mathrm{A}}\left(\epsilon_{\mathrm{F}}\right)+y N_{\mathrm{B}}\left(\epsilon_{\mathrm{F}}\right)
\end{aligned}
$$

Following the work of McMillan, ${ }^{8}$ we can write down the formula for $T_{c}$ :

$$
T_{c}=\frac{\Theta}{1,45} \exp -\frac{1.04\left(1+\lambda_{\text {eff }}\right)}{\lambda_{\text {eff }}-\mu_{\text {eff }}^{*}\left(1+0.62 \lambda_{\text {eff }}\right)}
$$

where the electron-phonon coupling constant

$$
\begin{aligned}
\lambda_{\text {eff }}= & \sum_{\alpha} \vec{t}^{2} \frac{d_{\alpha}^{2}}{d^{2}}\left\{x N_{\mathrm{A}}\left(\epsilon_{\mathrm{F}}\right) \tilde{D}_{\mathrm{A}}^{\alpha}\left[x q_{\mathrm{A}}^{2} N_{\mathrm{A}}\left(\epsilon_{\mathrm{F}}\right)+\frac{1}{4}\left(q_{\mathrm{A}}+q_{\mathrm{B}}\right)^{2} N_{\mathrm{B}}\left(\epsilon_{\mathrm{F}}\right)\right]\right. \\
& \left.+y N_{\mathrm{B}}\left(\epsilon_{\mathrm{F}}\right) \tilde{D}_{\mathrm{B}}^{\alpha}\left[y q_{\mathrm{B}}^{2} N_{\mathrm{B}}\left(\epsilon_{\mathrm{F}}\right)+\frac{1}{4} x\left(q_{\mathrm{A}}+q_{\mathrm{B}}\right)^{2} N_{\mathrm{A}}\left(\epsilon_{\mathrm{F}}\right)\right]\right\} \frac{1}{N\left(\epsilon_{\mathrm{F}}\right)}
\end{aligned}
$$

and the Coulomb pseudopotential

$$
\mu_{\text {eff }}^{*}=N\left(\epsilon_{\mathrm{F}}\right) U_{\text {eff }} /\left[1+N\left(\epsilon_{\mathrm{F}}\right) U_{\text {eff }} \ln \frac{W}{\Theta}\right]
$$

both depend on the alloy parameters, in particular on the concentration $x$, thus giving rise to a concentration dependence of the transition temperature $T_{c}$. The calculation of $T_{c}$ versus $x$ for various transition metal alloys will be the subject of a subsequent publication. 
In the above formulas $\Theta$ is of the order of the Debye temperature of the alloy, $W$ is the alloy bandwidth, ${ }^{25}$ and $\tilde{D}_{i}^{\alpha}$ denotes

$$
\tilde{D}_{i}^{\alpha}=-\frac{2}{\pi} \int_{-\infty}^{\infty} d \omega \frac{\operatorname{Im} D_{i}^{\alpha}(\omega+i \epsilon)}{\omega}, \quad i=\mathrm{A}, \mathrm{B}
$$

To obtain $T_{c}$ for various alloys one has to solve the CPA equation (48) and then calculate $N_{i}\left(\epsilon_{\mathrm{F}}\right)$ and $N\left(\epsilon_{\mathrm{F}}\right)$, then an equation similar to (48) for the calculation of the phonon Green's function $D(\omega)$ and then $\tilde{D}_{i}^{\alpha}{ }^{27,43}$

\section{DISCUSSION AND CONCLUSIONS}

We have developed a theory of strong coupling superconductivity in disordered transition metal alloys. The use of the alloy version of the BLF model ensures the proper treatment of the atomic character of the $d$ electrons responsible for superconductivity in such systems. We were able to obtain a closed set of equations determining the electron and phonon Green's functions and mass operators. These equations give a general microscopic description of an alloy in the spirit of the Migdal-Eliashberg approach. Written in a Wannier space, they refer to the fixed configuration of atoms in an alloy. Therefore an averaging is needed. This was performed in two different ways. First in section 3.1 we used a very simple approximation for the averaged Green's functions. As mentioned previously, this approximation gives a workable scheme for the derivation of the usual nonlinear Eliashberg equations written in terms of microscopic alloy parameters. The second approach is fully based on the CPA. We take into account the randomness not only through the parameters of the Hamiltonian but also in a self-consistent way through the configuration dependence of the single-site electron mass operator. Although similar to Refs. 25 and 26, our paper contains some further developments of these theories for strong coupling superconductivity in disordered alloys. Contrary to Ref. 25 , we take into account the effect of disorder on the phonon Green's function and we do not replace, as already mentioned, the single-site Green's functions $G_{\mathrm{A}}$ and $G_{\mathrm{B}}$ in the mass operator by the averaged Green's function $\bar{G}$. In Ref. 26 , the electron-phonon interaction Hamiltonian is not expressed in terms of microscopic parameters like $q_{0}^{i}, t_{i j}$, etc. The expression for the self-energy in Ref. 26 is limited to the contact model only, and in order to average the Green's function over the configuration a phenomenological ansatz is used for the anomalous self-energy. In contrast, we have derived the mass operator in a general way by means of the "irreducible" Green's functions, which permit the separation of the Hartree-FockBogolubov mean-field terms, and we have obtained an exact expression 
for the mass operator. It must be emphasized that for the random contact model limit we have derived and exploited the exact general relationship between the normal and anomalous parts of the CPA-averaged electron Green's function.

The present theory in its general form as well as the contact model version will be used in the near future for a discussion of the concentration dependence of $T_{c}$ in some transition metal alloys.

\section{ACKNOWLEDGMENTS}

The authors are grateful to Dr. N. M. Plakida, Dr. W. Kolley, and Prof. W. Weller for useful discussions.

\section{REFERENCES}

1. N. N. Bogolubov, Zh. Eksp. Teor. Fiz. 34, 58, 73 (1958).

2. N. N. Bogolubov, V. V. Tolmachev, and D. V. Shirkov, A New Method in the Theory of Superconductivity (Consultants Bureau, New York 1959).

3. N. N. Bogolubov, Usp. Fiz. Nauk 67, 549 (1959).

4. J. R. Schrieffer, Theory of Superconductivity (Benjamin, New York, 1964).

5. Y. Nambu, Phys. Rev. 117, 648 (1960).

6. G. M. Eliashberg, Zh. Eksp. Teor. Fiz. 38, 966 (1960) [Sov. Phys. JETP 11, 696 (1960)].

7. D. J. Scalapino, J. R. Schrieffer, and J. W. Wilkins, Phys. Rev. 148, 263 (1966).

8. W. L. McMillan, Phys. Rev. 167, 331 (1968).

9. P. W. Anderson, J. Phys. Chem. Solids 11, 26 (1959).

10. A. A. Abrikov, L. P. Gorkov, and I. E. Dzyaloshinski, Quantum Field Theory in Statistical Physics (Prentice Hall, Englewood Cliffs, New Jersey, 1963).

11. G. Bergmann, Phys. Rep. 27C, 161 (1976).

12. S. V. Vonsovskij, Yu. A. Izyumov, and E. Z. Kurmaev, Superconductivity of Transition Metals (Springer-Verlag, 1982).

13. P. B. Allen, in Dynamical Properties of Solids, G. W. Horton and A. A. Maradudin, eds. (North-Holland, Ämsterdam, 1980), p. 95.

14. H. Lustfeld, J. Low Temp. Phys. 12, 595 (1973).

15. K. Tankei and F. Takano, Prog. Theor. Phys. 51, 988 (1974).

16. G. Kerker and K. H. Bennemann, Solid State Commun. 14, 399 (1974).

17. A. Weinkauf and J. Zittartz, Solid State Commun. 14, 365 (1974).

18. A. Weinkauf and J. Zittartz, J. Low Temp. Phys. 18, 229 (1975).

19. O. A. Dubovsky, Fiz. Tverd. Tela (Leningrad) 20, 3119 (1978).

20. A. Beckmann, E. Kolley, and W. Kolley, Phys. Status Solidi (B) 10, K107 (1980).

21. E. Kolley, W. Kolley, and A. Beckmann, Phys. Status. Solidi. B 103, 775 (1981).

22. A. Beckmann, E. Kolley, and W. Kolley, Phys. Status Solidi. B 110, 557 (1982).

23. G. Grünewald and K. Scharnberg, Solid State Commun. 32, 955 (1979).

24. J. Appel, Phys. Rev. B 13, 3203 (1976).

25. G. Kerker and K. H. Bennemann, Solid State Commun. 15, 29 (1974).

26. H. Lustfeld, $Z$. Phys. 271, 229 (1974).

27. K. I. Wysokiński and A. L. Kuzemsky, Commun. JINR E17-81-614, Dubna (1981).

28. S. Barišić, J. Labbé, and J. Friedel, Phys. Rev. Lett. 25, 919 (1970).

29. A. L. Kuzemsky, A. Holas, and N. M. Plakida, Commun. JINR P17-82-493, Dubna (1982).

30. S. J. Poon, Solid State Commun. 18, 1489 (1976). 
31. C. M. Varma, E. I. Blount, P. Vashita, and W. Weber, Phys. Rev. B 19, 6130 (1979).

32. W. Weber, in Superconductivity in $d$ - and $f$-Band Metals, H. Suhl and M. B. Maple, eds. (Academic, New York, 1980), p. 131.

33. N. M. Plakida, J. Phys. C 4, 1680 (1971).

34. A. L. Kuzemsky, Theor. Math. Phys. 36, 208 (1978).

35. G. M. Vujičić, A. L. Kuzemsky, and N. M. Plakida, Theor. Math. Phys. 53, 138 (1982).

36. N. N. Bogolubov and S. V. Tyablikov, Sov. Phys. Dok. 4, 604 (1959).

37. D. N. Zubarev, Sov. Phys. Usp. 3, 320 (1960).

38. K. Machida, Prog. Theor. Phys. 66, 41 (1981).

39. J. A. Blackman, D. M. Esterling, and N. F. Berk, Phys. Rev. B 4, 2412 (1971).

40. H. Katayama and J. Kanomori, J. Phys. Soc. Jpn. 45, 1157 (1978).

41. B. Velicky, S. Kirkpatrick, and H. Ehrenreich, Phys. Rev. 175, 747 (1968).

42. P. Soven, Phys. Rev. 156, 809 (1967).

43. D. W. Taylor, Phys. Rev. 156, 1017 (1967). 